\title{
The measurement of velocity gradients in laminar flow by homodyne light-scattering spectroscopy
}

\author{
By G. G. FULLER, J. M. RALLISON†, R. L. SCHMIDT \\ AND L. G. LEAL \\ Department of Chemical Engineering, \\ California Institute of Technology, Pasadena, California 91125
}

(Received 5 March 1979 and in revised form 12 March 1980)

\begin{abstract}
A technique for measuring velocity gradients in laminar flows by homodyne light scattering is presented. A theory which describes the light-scattering spectrum is derived that includes the effects of different types of linear flow fields, particle diffusion and the intensity profile in the scattering volume. The conditions which must be satisfied in order that the theory describe the experimental situation are outlined and complementary experiments are performed which both verify the theory and apply the technique. Verification is provided using the flow in a Couette device, and the flow due to single rotating cylinder in a large bath of fluid. The technique is then applied to measure the spatial variation of the shear rate in a four-roll mill.
\end{abstract}

\section{Introduction}

The development of an accurate experimental method for determination of velocity gradients in a liquid undergoing steady, laminar flow is a problem of obvious practical importance. There is, of course, a variety of experimental techniques to determine the velocity in a flowing fluid, one of the most popular methods being laser-Doppler velocimetry. The use of laser light as a probe has the distinct advantage that it imparts essentially no disturbance to the velocity field. However, relatively little has been done which addresses the direct determination of velocity gradients.

The usual procedure for measuring velocity gradients using the laser-Doppler velocimeter ( $\mathrm{LDV})$ is to first measure the velocity as a function of spatial position and then differentiate the data. However, this procedure suffers from the inherent difficulties of differentiating experimental data and is particularly suspect in the regions of large velocity gradients which are likely to be of greatest interest. Not only may the region being studied be too thin to allow sufficient spatial resolution of the velocity field for differentiation with any reasonable accuracy (the LDV system yields an average velocity over the scattering volume which is typically about $0.1 \mathrm{~mm}$ in linear extent), but the LDV signal is also complicated by a large variation in velocity across the scattering volume and is difficult to interpret (Berman \& Dunning 1973). In this paper, we demonstrate how the homodyne mode of light scattering spectroscopy may be used to directly measure the average velocity gradient in the scattering volume from a single in situ experiment, thereby minimizing both of these problems. This

$\dagger$ Department of Applied Mathematics and Theoretical Physics, University of Cambridge.

$\ddagger$ Chevron Oil Field Research, La Habra, California. 
technique has been largely overlooked as a way to probe flowing liquids, although it was one of the earliest methods to be used which incorporated a laser light source. The earliest application was by Bourke et al. (1970), who studied a turbulent pipe flow using homodyne spectroscopy and determined that spatial variations in the velocity existing inside the scattering volume were the dominant contribution to the frequency spectrum of the scattered light. The use of this technique for the study of turbulent flow fields is, however, greatly limited owing to a lack of knowledge of the nature of the kinematics of the flow field. It is, therefore, necessary to adopt some model of the turbulent flow in order to analyse the experimental data and for that reason the interpretation is somewhat equivocal and dependent on the quality of the model used. This paper treats the much simpler case of steady, laminar flow where the kinematics are known and there is therefore no need for recourse to modelling of the flow. The analysis of the experiment is consequently less ambiguous.

The paper consists of two main parts. In the first, we present the theoretical developments which are necessary to determine the local velocity gradient from the homodyne spectrum of light scattered from small particles which are immersed in a steady laminar flow. In the second, we report complementary experimental results which verify the method and illustrate its application. A primary objective of both the analysis and the experiments is to delineate the conditions which must be satisfied in order that the measurement of velocity gradients by homodyne light scattering be accurate and unambiguous. For this reason, our theoretical analysis considers not only the effect of a velocity gradient on the observable frequency spectrum, but also the effects of different types of laminar motion, of variations in the light intensity profile of the incident beam in the scattering volume, and of random, diffusive motions of the scattering particles.

\section{Theory}

We consider the light seattered by small particles which are immersed in a liquid that is undergoing an arbitrary steady, laminar motion. The light-scattering experiment is sketched in figure 1 . The incident light is a single, monochromatic beam which is generated by a laser source, and the scattered light is viewed by a square-law detector (in our case, a photomultiplier tube) through two pinholes which serve to collimate the scattered beam, and thus define the extent of the scattering volume. The particles are assumed to be rigid, and small enough that their mean translational velocity is indistinguishable from the 'undisturbed' velocity of the suspending liquid. Furthermore, they are assumed to be present at low concentration so that they neither alter the motion of the suspension as a whole, nor contribute to a significant level of multiple-scattering events. Finally, the Rayleigh-Debye condition (Berne \& Pecora 1976) is assumed to be satisfied, which requires an upper bound of $\lambda / 4 \pi|m-1|$ on the average particle dimension, where $\lambda$ is the wavelength of the incident light and $m$ is the ratio of the solvent refractive index to that of the particles.

The frequency spectrum of the scattered light from the scattering volume $V$ is determined by the motions of the scattering elements (both random and deterministic). By measuring the spectrum of the scattered light mixed with the incident light (heterodyne mode) it is possible to determine the mean velocity of the scattering centres in the scattering volume (Edwards et al. 1971). The effect of velocity gradients and of random motions due to diffusion is to broaden the heterodyne spectrum, but 


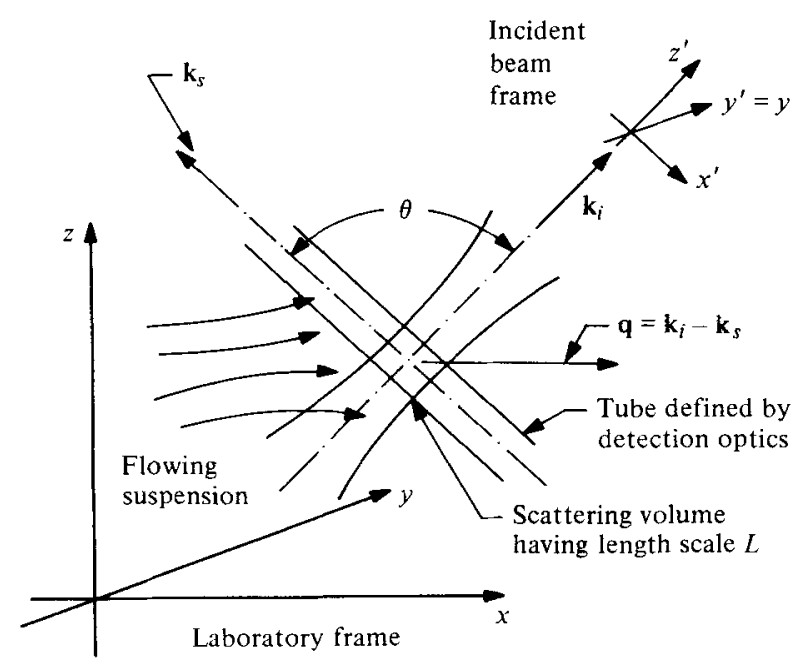

FIGURE 1. Light-scattering geometry: $\mathbf{k}_{s}$ is the scattered-light wave vector, $\mathbf{k}_{i}$ is the incidentlight wave vector, $\theta$ is the scattering angle. The vector $\mathbf{q}$ is oxientated along the $x$ axis in the laboratory frame.

these are second-order effects compared to the Doppler shift of the frequency which is caused by the mean motion of the particles in suspension.

The present paper is concerned with the frequency spectrum of scattered light when it is 'mixed with itself' (i.e. the so-called homodyne mode). In this case, it will be seen that the effect of the mean velocity on the frequency correlation function can be neglected, relative either to the effects of differences in the mean velocity across the scattering volume or of particle diffusion, provided only that the time scales associated with the mean velocity gradient, or with diffusion on the length scale $|\mathbf{q}|^{-1}$ (see, for example, figure 1) are sufficiently short compared to the transit time of a particle across the scattering volume. In these circumstances, we shall see that the homodynescattering experiment provides a direct measurement of either the mean velocity gradient or of the particle diffusivity, depending upon which of these dynamical processes is characterized by the shorter time scale.

The scattered light spectrum for the heterodyne mode is given by the heterodyne correlation function, $F_{1}$, which, in the time domain has the form (Berne \& Pecora 1976)

$$
F_{1}(\mathbf{q}, t)=\sum_{j=1}^{N}\left\langle E_{j}^{*}(0) E_{j}(t) \exp \left\{i \mathbf{q} \cdot\left[\mathbf{r}_{j}(t)-\mathbf{r}_{j}(0)\right]\right\}\right\rangle .
$$

Here, $\mathbf{q}$ is the scattering vector which is defined in figure $1, E_{j}(t)$ is the amplitude of the light scattered by particle $j$ and $\mathbf{r}_{j}(t)$ is the position of the centroid of particle $j$ with respect to an arbitrary origin in the scattering volume. The summation is over all particles in the scattering volume for a given realization of the scattering experiment and the angle brackets represent an ensemble average over many realizations.

Provided that the contribution of each particle to the scattered light is statistically independent of any other and randomly distributed, it may be shown (Berne \& Pecora 1976 ) that the homodyne spectrum, $F_{2}$, is related to the heterodyne spectrum by

$$
F_{2}(\mathbf{q}, t) \equiv\left|F_{1}(\mathbf{q}, t)\right|^{2} .
$$


It can be shown that this relation is also valid for the case of flowing particles where the deterministic motions dominate those due to random processes, provided the time scale associated with the decay of the heterodyne correlation function is sufficiently short compared to the reciprocal of the local velocity gradient $\gamma^{-1}$. This condition ensures that during the time scale of the experiment, any two particles will be statistically independent allowing the homodyne correlation function to be factored into the square modulus of the heterodyne correlation function as in equation (2). This condition is almost always satisfied under normal laboratory conditions. Thus, we shall first analyse the heterodyne spectrum via equation (1), and then use (2) to calculate the corresponding homodyne spectrum.

Equation (1) can be simplified if the amplitude function $E_{j}(t)$ and the phase factor $\exp (i \mathbf{q} .[\mathbf{r}(t)-\mathbf{r}(0)])$ vary on sufficiently different time scales. For spherical, optically homogeneous particles the amplitude function varies principally with the transit time of a particle across the scattering volume. If the particles are optically or geometrically anisotropic, the amplitude function will also vary on the additional time scale associated with particle rotation. However, we shall focus our attention for the moment on the simpler case of particles which are isotropic scatterers and return to the question of anisotropy at the end of the section. Thus, the amplitude factor will be assumed to vary on the transit time scale

$$
\tau_{t}=L / U
$$

where $L$ is a length scale characteristic of the scattering volume, and $U$ is the mean particle velocity in the volume. The phase factor, on the other hand, varies principally on the shortest of the possible time scales which is characteristic of particle displacement over a length scale, $|\mathbf{q}|^{-1}$. As we shall show later, this is usually

$$
\tau_{s} \equiv(q \gamma L \cos \phi)^{-1}
$$

which is inversely proportional to the magnitude of the local velocity gradient. Here, $\phi$ is the angle between the scattering vector, $\mathbf{q}$, and the local mean velocity vector, $\mathbf{v}$. In order to minimize $\tau_{s}$, this angle will normally be set to zero in the present application. Now, a typical magnitude for $|\mathbf{q}|$ is $10^{5} \mathrm{~cm}^{-1}$. Thus, by controlling the size of the scattering volume, $L$, the time scale, $\tau_{s}$, of variations in the phase factor of (1) can be made quite short [say, $O\left(10^{-2}\right)$ or smaller] relative to the time scale $\tau_{t}$, of variations in the amplitude factor for most systems. We shall therefore assume the condition

$$
\tau_{t} / \tau_{s}=q \gamma L^{2} / U \gg 1
$$

to hold, thus implying that the particle moves only a short distance relative to $L$ on the time scale $\tau_{s}$. Under these circumstances, the amplitude factor in (1) is essentially constant over the time scale for decay of the correlation function (i.e. $\tau_{s}$ ), and equation (1) can be approximated in the simpler form

$$
F_{1}(\mathbf{q}, t)=\sum_{j=1}^{N} I_{j}\left\langle\exp \left\{i \mathbf{q} \cdot\left[\mathbf{r}_{j}(t)-\mathbf{r}_{j}(0)\right]\right\}\right\rangle=\sum_{j=1}^{N} I_{j} F_{s j}(\mathbf{q} t)
$$

where $I_{j} \equiv\left\langle E_{j}(0)^{*} E_{j}(0)\right\rangle$ is the intensity of light seattered by particle $j$ and $F_{s j}$ is the so-called self-intermediate scattering function. We may note that for intermediate values of $\tau_{t} / \tau_{s}$, i.e. $\leqslant 1$, the phase and amplitude factors in (1) vary on comparable 
time scales, and experimental analysis of the frequency spectrum is impractical except in the opposite limit $\tau_{t} / \tau_{s} \ll 1$, where measurement of $F_{1}(\mathbf{q}, t)$ corresponds to the single beam velocimeter, described by Pike (1976).

We shall restrict our attention in the present communication to the limiting case (5) where the approximation (6) is valid. Further analysis of the correlation function thus reduces to a determination of the self-intermediate scattering function, $F_{s j}(\mathbf{q}, t)$. This analysis is facilitated by first solving for the Fourier transform of $F_{s j}$, (the VanHove self space-time correlation function)

$$
\begin{aligned}
G_{s j} & \equiv \int d^{\mathbf{3}} \mathbf{q} \exp \left[-i \mathbf{q} \cdot \tilde{\mathbf{x}}_{j}\right]\left\langle\exp \left\{i \mathbf{q} \cdot\left[\mathbf{r}_{j}(t)-\mathbf{r}_{j}(0)\right]\right\}\right\rangle \\
& =\left\langle\delta\left(\tilde{\mathbf{x}}_{j}-\left[\mathbf{r}_{j}(t)-\mathbf{r}_{j}(0)\right]\right)\right\rangle,
\end{aligned}
$$

and then inverting to determine $F_{s j}$. It may be seen from (7) that $G_{s j}$ is nothing more than the conditional probability density for a particle to be at a position $\mathbf{r}_{j}(t)$ at a time, $t$, given that it starts at $\mathbf{r}_{j}(0)$ at $t=0$ (Berne \& Pecora 1976). Thus, $G_{s j}$ represents the solution of the classical convective diffusion equation for the particle,

subject to

$$
\frac{\partial G_{s j}}{\partial t}+\nabla \cdot\left(\mathrm{V}_{j} G_{s j}\right)-D \nabla^{2} G_{s j}=0
$$

$$
G_{s j}\left(\tilde{\mathbf{x}}_{j}, 0\right)=\delta\left(\tilde{\mathbf{x}}_{j}\right)
$$

Here, it should be emphasized that $\tilde{\mathbf{x}}_{j}$ is defined relative to a co-ordinate system with an origin at $\mathbf{r}_{j}(0)$. Once $G_{s j}$ is known, the function $F_{s j}$ can be determined by means of the inverse transform

$$
F_{s j}(\mathbf{q}, t) \equiv \frac{1}{(2 \pi)^{\frac{8}{2}}} \int d^{3} \mathbf{x} \exp \left[i \mathbf{q}, \tilde{\mathbf{x}}_{j}\right] G_{s j}\left(\tilde{\mathbf{x}}_{j}, t\right) .
$$

The diffusion coefficient, $D$, has been taken as isotropic in (8) as a consequence of the earlier restriction to spherical particles.

Since the convection or diffusion process relevant to the light-scattering experiment is characterized by a maximum length scale, $L$, corresponding to the scattering volume, we can approximate the velocity of particle $j$ as

$$
\mathbf{V}_{j}=\overline{\mathbf{V}}_{j}+\boldsymbol{\Gamma} \cdot \overline{\mathbf{x}}_{j}
$$

where $\overline{\mathbf{V}}_{j}$ is the velocity of a particle in the scattering volume at the point $\mathbf{x}=\mathbf{r}_{j}$ and $\boldsymbol{\Gamma}$ is the local velocity gradient tensor. Substituting (9) into (8) and taking the Fourier transform thus leads to

$$
\left.\begin{array}{c}
\frac{\partial F_{s j}}{\partial t}-i \overline{\mathbf{V}}_{j} \cdot \mathbf{q} F_{s j}-\mathbf{q} \cdot \mathbf{\Gamma} \cdot \nabla_{q} F_{s j}+D q^{2} F_{s j}=0, \\
F_{s j}(\mathbf{q}, 0)=\frac{1}{(2 \pi)^{\frac{3}{2}}} .
\end{array}\right\}
$$

The solution of (10) can be obtained by the method of characteristics. The result is

$$
\left.\begin{array}{c}
F_{s j}(\mathbf{q}, t)=\frac{1}{(2 \pi)^{\frac{3}{2}}} \exp \left\{-\int_{0}^{t} d t^{\prime}\left[D q^{\prime 2}\left(t^{\prime}\right)+i \dot{\overline{\mathbf{V}}}_{j} \cdot \mathbf{q}^{\prime}\left(t^{\prime}\right)\right]\right\} \\
\frac{d \mathbf{q}^{\prime}}{d t}=-\boldsymbol{\Gamma}^{T} \cdot \mathbf{q}^{\prime}, \quad \mathbf{q}^{\prime}(0)=\mathbf{q} .
\end{array}\right\}
$$


In order to calculate the heterodyne correlation function $F_{1}(\mathbf{q}, t)$, it is necessary to sum over all the particles in the scattering volume. Provided the number of such particles is large, this sum can be transformed to a volume integral giving

$$
F_{1}(\mathbf{q}, t)=\iiint d^{3} x I(\mathbf{x}) \exp \left\{-\int_{0}^{t} d t^{\prime}\left[D q^{\prime 2}\left(t^{\prime}\right)+i \bar{V}(\mathbf{x}) \cdot \mathbf{q}^{\prime}\left(t^{\prime}\right)\right]\right\}
$$

where $I(\mathbf{x})$ is the intensity profile of the incident beam in the scattering volume, and $\mathrm{V}(x)$ is the velocity of the particles, which is approximated as

$$
\mathbf{V}(\mathbf{x})=\mathbf{U}+\mathbf{\Gamma} \cdot \mathbf{x} .
$$

Here $U$ is the average particle velocity within the scattering volume, rather than the mean velocity of the position of particle $j$ at time zero as in (9). Substituting (13) into (12), the heterodyne correlation function then becomes

$$
\begin{aligned}
F_{1}(\mathbf{q}, t) & =\exp \left\{-\int_{0}^{t}\left[D q^{\prime 2}\left(t^{\prime}\right)+i \mathbf{U} \cdot \mathbf{q}^{\prime}\left(t^{\prime}\right)\right] d t^{\prime}\right\} \iiint d x^{3} I(\mathbf{x}) \exp \left\{-\int_{0}^{t} d t^{\prime} \mathbf{q}^{\prime}\left(t^{\prime}\right) \cdot \mathbf{\Gamma} \cdot \mathbf{x}\right\} \\
& =\exp \left\{-\int_{0}^{t}\left[D q^{\prime 2}\left(t^{\prime}\right)+i \mathbf{U} \cdot \mathbf{q}^{\prime}\left(t^{\prime}\right)\right] d t^{\prime}\right\} I\left(\int_{0}^{t} d t^{\prime} \mathbf{q}^{\prime}\left(t^{\prime}\right) \cdot \Gamma\right) .
\end{aligned}
$$

The corresponding homodyne correlation function, calculated from equation (2), is then

$$
\begin{aligned}
F_{2}(\mathbf{q}, t) & =\exp \left\{-2 \int_{0}^{t} d t^{\prime} D q^{\prime 2}\left(t^{\prime}\right)\right\}\left|\iiint d x^{3} I(x) \exp \left\{-i \int_{0}^{t} d t^{\prime} \mathbf{q}^{\prime}\left(t^{\prime}\right) \cdot \mathbf{\Gamma} \cdot \mathbf{x}\right\}\right|^{2} \\
& =\exp \left\{-2 \int_{0}^{t} d t^{\prime} D q^{\prime 2}\left(t^{\prime}\right)\right\}\left|I\left(\int_{0}^{t} d t^{\prime} \mathbf{q}^{\prime}\left(t^{\prime}\right) \cdot \mathbf{\Gamma}\right)\right|^{2}
\end{aligned}
$$

where $I$ is the Fourier transform of the intensity profile $I$, and

$$
\frac{d \mathbf{q}^{\prime}}{d t}=-\Gamma^{T} \cdot \mathbf{q}^{\prime}, \quad \mathbf{q}^{\prime}(0)=\mathbf{q}
$$

The homodyne correlation function differs from the heterodyne function primarily in the fact that there is no dependence on the mean velocity $U$. In particular, both functions involve the velocity gradient, $\boldsymbol{\Gamma}$, and both therefore could, in principle, provide a basis for its experimental determination. As a practical matter, however, the homodyne mode provides a much superior technique. The response of the heterodyne correlation function with time will, in most cases, be dominated by the mean velocity term since the associated time scale, $(q U)^{-1}$ will usually be much shorter than either of the time scales, $\left(D q^{2}\right)^{-1}$ or $(q \gamma L)^{-1}$, which are associated with diffusion or the velocity gradient, $\|\boldsymbol{\Gamma}\|=\gamma$. Thus, except for extremely small values of $U$, determination of the velocity gradient from the heterodyne correlation function would be difficult. The magnitude of the homodyne correlation, on the other hand, will normally be dominated by the velocity gradient term. Furthermore, the homodyne technique is much simpler to implement since no additional optics or alignment is required to deflect and mix the incident beam with the scattered signal.

The effect of the velocity gradient enters primarily through the Fourier transform,

$$
I\left(\int_{0}^{t} d t^{\prime} \mathbf{q}^{\prime}\left(t^{\prime}\right) \cdot \Gamma\right)
$$


of the intensity profile of the incident beam in the scattering volume. By way of example, we have calculated the complete homodyne correlation function for two specific linear flows of the type (13). The first is simple shear flow where

and

$$
\Gamma=\gamma\left(\begin{array}{lll}
0 & 1 & 0 \\
0 & 0 & 0 \\
0 & 0 & 0
\end{array}\right)
$$

$$
F_{2}(\mathbf{q}, t)=\exp \left\{-2 D\left[k^{2}\left(1+\frac{1}{3}(\gamma t)^{2}\right)+\gamma t . k l+l^{2}+m^{2}\right] t\right\}\left|\iiint d^{3} \mathbf{x} I(\mathbf{x}) \exp \{-i \gamma k y t\}\right|^{2},
$$

and the second is plane, hyperbolic flow where

and

$$
\Gamma \equiv \gamma\left(\begin{array}{rrr}
1 & 0 & 0 \\
0 & -1 & 0 \\
0 & 0 & 0
\end{array}\right)
$$

$$
\begin{aligned}
F_{2}(\mathbf{q}, t)=\exp \{ & \left.-2 D\left[k^{2}\left(\frac{e^{2 \gamma t}-1}{2 \gamma}\right)+l^{2}\left(\frac{1-e^{-2 \gamma t}}{2 \gamma}\right)+m^{2} t\right]\right\} \\
\times & \left|\iiint d^{3} \mathbf{x} I(\mathbf{x}) \exp \left\{-i k x\left(1-e^{\gamma t}\right)\right\} \exp \left\{i l y\left(1-e^{-\gamma t}\right)\right\}\right|^{2} .
\end{aligned}
$$

The components of $q$ have been denoted as $(k, l, m)$ in (16) and (17). Examination of these expressions shows that the homodyne correlation function varies on three independent time scales

$$
\tau_{f} \equiv \gamma^{-1}, \quad \tau_{D} \equiv\left(q^{2} D\right)^{-1}
$$

and $\tau_{s}(\phi \equiv 0)$, which was defined in equation (4). Homodyne light scattering spectroscopy is practical as a means for determining the local velocity gradient, only when

$$
\tau_{s} \ll\left(\tau_{f}, \tau_{D}\right) .
$$

In this case, the expressions (16) and (17) can be approximated in the simpler forms

and

$$
F_{2}(\mathbf{q}, t) \sim\left|\iiint d^{3} \mathbf{x} I(\mathbf{x}) \exp \{-i k \gamma y t\}\right|^{2}
$$

$$
F_{2}(\mathbf{q}, t) \sim\left|\iiint d^{3} \mathbf{x} I(\mathbf{x}) \exp \{-i \gamma k x t+i \gamma l y t\}\right|^{2}
$$

respectively, and the time rate of decay of the correlation function provides a direct measure of the velocity gradient. In the case of a general linear flow subject to the same assumptions we obtain

$$
F_{2}(\mathbf{q}, t)=\left|\iiint d^{3} \mathbf{x} I(\mathbf{x}) \exp \{-i \mathbf{q} \cdot \mathbf{\Gamma} \cdot \mathbf{x} t\}\right|^{2} .
$$

The expressions (19)-(21) provide the theoretical basis for determining the velocity gradient from a measurement of the homodyne correlation function, and will be used 
to analyse the experimental data of $\S 3$ of this paper. If either of the conditions (18), or (5) is not satisfied for a given experimental set-up, the homodyne spectrum will reflect (or even be dominated by) additional contributions which arise from particle diffusion and/or translation across the scattering volume. In this case, the utility of homodyne scattering as a tool for determination of the local velocity gradient will be lost. Because of the importance of the relative magnitudes of the various time scales, $\tau_{f}, \tau_{s}$ and $\tau_{D}$, in establishing the domain of validity of the homodyne experiment as a method of measuring the velocity gradient, it is worthwhile to consider their relative values for typical experimental conditions. Thus, we have

and

$$
\frac{\tau_{f}}{\tau_{s}}=q l\left(\frac{L}{l}\right)
$$

$$
\frac{\tau_{D}}{\tau_{s}}=\frac{P e}{q l}\left(\frac{L}{l}\right)
$$

where $l$ is the characteristic length scale of the particle and $P e \equiv \gamma l^{2} / D$ is the particle Péclet number. Now, the ratio $(L / l)$ will typically be $O\left(10^{3}\right)$ or larger, whereas $q l$ is generally $O(1)$ or less in order that the Rayleigh-Debye approximation be valid. Thus, $\tau_{f} / \tau_{s} \sim O\left(10^{3}\right)$, while $\tau_{D} / \tau_{s} \sim 10^{3} \mathrm{Pe}$. The latter ratio, $\tau_{D} / \tau_{s}$, will be large provided $P e>10^{-1}$ to $10^{-2}$. For non-zero $\gamma$, this condition on $P e$ will almost always be satisfied. Thus, the condition (18) will be satisfied and the homodyne scattering experiment will almost always be dominated by the contribution of the velocity gradient in the form (19)-(21).

To complete the determination of the expected homodyne spectrum, when the conditions (5) and (18) are met, it is necessary to calculate the Fourier transform, (21), of the intensity distribution of the incident beam in the scattering volume. In general, however, this intensity profile will not be precisely known, $\dagger$ and it is thus important, if homodyne scattering is to be effective for determination of the local velocity gradient, that the spectrum be relatively insensitive to moderate variations in $I(\mathbf{x})$. For purposes of investigating this sensitivity, we have considered the following two intensity distributions for the beam geometry of figure 1 with $\mathbf{q}=(q, 0,0)$

$$
I^{(1)}\left(x^{\prime} y^{\prime} z^{\prime}\right)=\exp \left[-\left(x^{\prime 2}+y^{\prime 2}\right) / L^{2}-z^{\prime 2} / \alpha^{2} L^{2} \operatorname{cosec}^{2} \theta\right]
$$

and the 'tophat' profile

$$
I^{(2)}\left(x^{\prime} y^{\prime} z^{\prime}\right)=\left\{\begin{array}{lll}
\exp \left(-z^{\prime 2} / \alpha^{2} L^{2} \operatorname{cosec}^{2} \theta\right) & \text { for } & \left(x^{\prime 2}+y^{\prime 2}\right) \leqslant L^{2} \\
0 & \text { for } & \left(x^{\prime 2}+y^{\prime 2}\right) \geqslant L^{2}
\end{array}\right.
$$

where $\theta$ is the scattering angle, $\mathbf{x}^{\prime}=\left(x^{\prime}, y^{\prime}, z^{\prime}\right)$ is the beam co-ordinate system defined in figure $1, L$ is the length-scale characteristic of the width of the incident beam and $\alpha$ is the ratio of the length of the scattering volume in the $z$ direction to the width $L$. With these expressions for the intensity profile, the equations (19) and (20) yield the following. For shear flow, q. $\mathbf{\Gamma} \cdot \mathbf{x} t=q \gamma y t$ :

(a) intensity profile $I^{(1)}: F_{2}(\mathbf{q}, t)=\exp \left\{-\frac{1}{2} q^{2} \gamma^{2} L^{2} t^{2}\right\}$;

† In spite of the fact that $I(\mathbf{x})$ can be measured in a reasonably straightforward manner, it will only stay fixed for about 24 hours, and accurate knowledge of $I(\mathbf{x})$ would thus require constant recalibration of the instrument. 


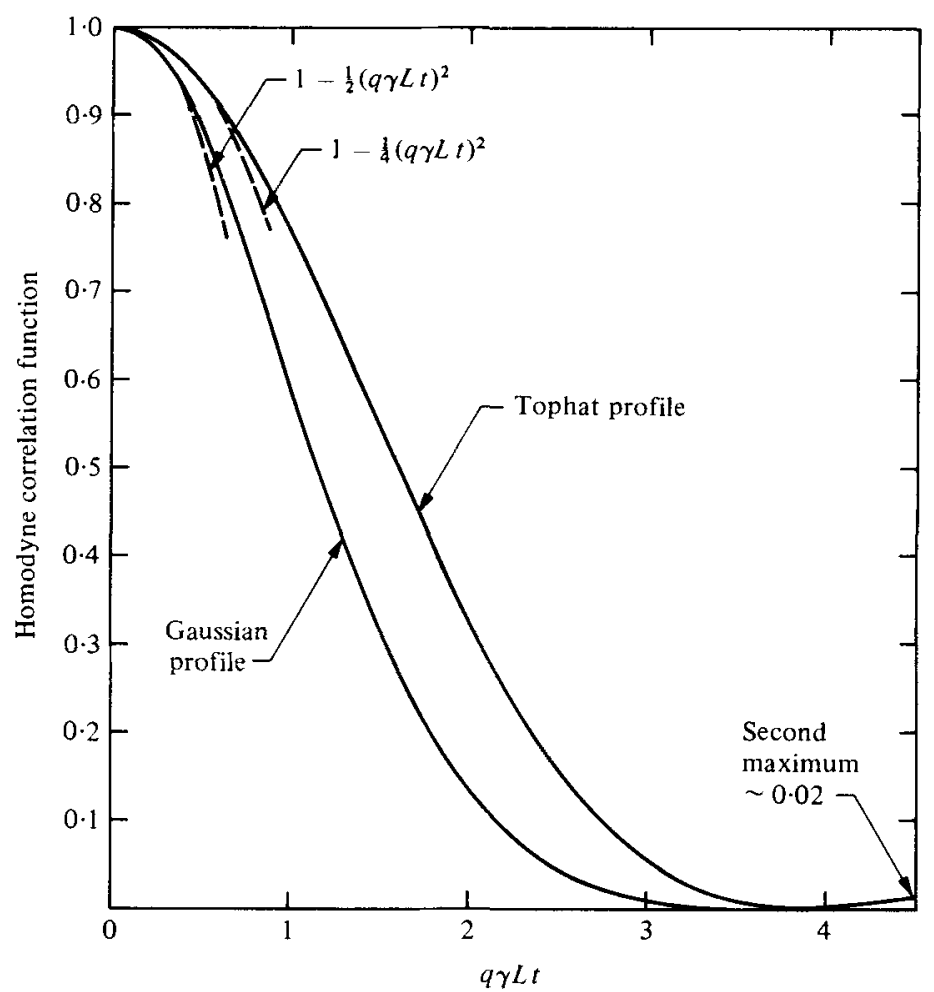

Figure 2. Calculated homodyne correlation function for two choices of the light-intensity profile in the scattering volume, using the scattering geometry of figure 1.

(b) intensity profile $I^{(2)}: F_{2}(\mathbf{q}, t)=\left[2 J_{1}(q \gamma L t) / q \gamma L t\right]^{2}$.

For two-dimensional extensional flow, $\mathbf{q} \cdot \mathbf{\Gamma} \cdot \mathbf{x} t=q x \gamma t=q z^{\prime} \sin \frac{1}{2} \theta \gamma t+q x^{\prime} \cos \frac{1}{2} \theta \gamma t$ :

(a) intensity profile $I^{(1)}: F_{2}(\mathbf{q}, t)=\exp \left[-\frac{1}{2} q^{2} \gamma^{2} L^{2} t^{2}\left(\cos ^{2} \frac{1}{2} \theta+\alpha^{2} \sin ^{2} \frac{1}{2} \theta / \sin ^{2} \theta\right)\right]$

(b) intensity profile $I^{(2)}: F_{2}(\mathbf{q}, t)=\exp \left[-\frac{q^{2} \gamma^{2} L^{2} \alpha^{2} \sin ^{2} \frac{1}{2} \theta}{2 \sin ^{2} \theta}\right]\left[\frac{2 J_{1}\left(q \gamma L t \cos \frac{1}{2} \theta\right)}{q \gamma L t \cos \frac{1}{2} \theta}\right]^{2}$.

Here $J_{1}$ is a Bessel function of the first kind.

Figure 2 compares the correlation functions described by equations (24) and (25). For large times, the behaviour of the correlation function, $F_{2}(\mathbf{q}, t)$, is fundamentally different for the two intensity distribution functions: $I^{(1)}$ produces monotonically decreasing correlation functions while those calculated for $I^{(2)}$ show time-dependent oscillations. However, the characteristic time scale in both cases is proportional to $q \gamma L$ and in the region of primary experiment interest $(q \gamma L t \leqslant 4)$ the qualitative behaviour produced by both $I^{(1)}$ and $I^{(2)}$ is the same. Comparison of (24) and (25) or (26) and (27) suggests that the principal influence of the flow type on $F_{2}(q t)$ is to alter the dependence of the characteristic decay time on the scattering angle $\theta$.

In general, the correlation function will be a function of more than one component of the velocity gradient tensor $\Gamma$, which has eight independent components if the fluid is incompressible. For the two specific flow types considered above and in the experiments described in the following section, the orientation of the vector $\mathbf{q}$ was chosen 
so that only one component of $\boldsymbol{\Gamma}$ was projected on to $\mathbf{q}$. This will not in general be possible. However, the individual components of $\boldsymbol{\Gamma}$ can still be measured, at least in principle, by creating an anisotropic scattering volume which has one length scale which is considerably larger than the other two. Thus, for example, we may consider an incident intensity distribution

$$
I(\mathbf{x})=\delta(x) \delta(y) f(z),
$$

where $\delta()$ is a Dirac delta function, in which it is assumed that the length scale of the scattering volume in the $z$ direction is much larger than along $x$ or $y$. In this case, the gradients in the velocity with respect to $z$ will dominate the decay of the correlation function and each individual component of $\boldsymbol{\Gamma}$ can be measured by independently varying the orientations of the scattering vector, $\mathbf{q}$, and the vector defining the largest length scale of the scattering volume.

All of the analysis of this section, from equation (3) onward, has been restricted to spherical particles, which are optically homogeneous and isotropic. If the particles are non-spherical or optically anisotropic, their rotational motions will influence the light-scattering spectrum, both through the amplitude function and the phase factor of equation (1). The time scale of rotationally induced variations in the amplitude factor will be $\Omega^{-1}$ where $\Omega$ is the magnitude of the angular velocity of the particle. In order to consider the role of rotations on the phase factor it is necessary to include an integration over the internal co-ordinates of equation (11). For a rod-like particle of length $l$ and unit vector $\mathbf{p}(t)$ along the rod axis, the homodyne correlation function (neglecting diffusive motions) is

$$
\begin{aligned}
F_{2}(\mathbf{q}, t)=\left|\iiint d^{3} x I(\mathbf{x}) \exp \{i \mathbf{q} \cdot \mathbf{\Gamma} \cdot \mathbf{x} t\}\right|^{2} \\
\times\left|\iiint_{-l / 2}^{l / 2} \exp \left\{i \mathbf{q} \cdot\left(r^{\prime} \mathbf{p}(t)-r^{\prime \prime} \mathbf{p}(0)\right)\right\} d r^{\prime} d r^{\prime \prime} d^{2} \mathbf{p}(0)\right|^{2} .
\end{aligned}
$$

Thus, the time scale of variations in the phase factor which are caused by particle rotation is

$$
t_{I}=(q l \Omega)^{-1}
$$

Since the angular velocity $\Omega$ will be at most the same order as the velocity gradient $\gamma$, the time scales characteristic of rotations of anisotropic particles will in general be long compared to the velocity gradient time scale $(q \gamma L)^{-1}$ and so may be ignored.

The analysis can also be extended to include the effects of curvature in the velocity field $\partial^{2} v_{i} / \partial x_{j} \partial x_{k}$. This may be important if there is a significant change in the velocity gradient over the scattering volume. For example, in the case of shear flow already considered, if the velocity field is actually

$$
\mathbf{v}=\left(U+\gamma y+\beta y^{2}, 0,0\right)
$$

the homodyne correlation function becomes (using the Gaussian intensity distribution $I^{(1)}$ and the geometry of figure 1)

$$
\begin{aligned}
F_{2}(\mathbf{q}, t) & =\left|\iiint d^{3} x^{\prime} I^{(1)}\left(\mathbf{x}^{\prime}\right) \exp \left\{i q \gamma y t+i \beta q y^{2} t\right\}\right|^{2} \\
& =\left(\frac{\pi}{1+q^{2} \beta^{2} L^{4} t^{2}}\right)^{\frac{1}{2}} \exp \left[-\frac{q^{2} \gamma^{2} L^{2} t^{2}}{2\left(1+q^{2} \beta^{2} L^{4} t^{2}\right)}\right] .
\end{aligned}
$$


If $\beta^{2} L^{2} / \gamma^{2} \ll 1$, the curvature effect will be unimportant and this expression reduces to the form (23) found earlier for a linear shear field. If this condition is not satisfied, on the other hand, the homodyne correlation function is no longer the square modulus of the Fourier transform of the intensity function and a new time scale $\left(q \beta L^{2}\right)^{-1}$ is introduced. Experimentally, the generally undesirable effects of curvature in the velocity field could thus be detectable, in principle, by the presence of an algebraic, rather than exponential, decay in the correlation function with a $t^{-1}$ dependence if the intensity distribution is Gaussian.

\section{Experimental}

In the preceding section, we have considered theoretically the light-scattering correlation function for the classical homodyne-scattering experiment applied to a flowing suspension. We have shown that the dominant contribution, under normal circumstances, will derive from the mean velocity gradient in the scattering volume, thus suggesting the potential of applying the homodyne scattering experiment to the technological problem of actually measuring the magnitude of-velocity gradients in flowing liquids.

In this section, we report the results of some exploratory experiments which were designed to test the validity of our theoretical results, and to explore the utility of homodyne light scattering as a method of measuring velocity gradients. Thus, two sets of flow experiments were performed under conditions, discussed in the preceding section, where the homodyne correlation function should decrease exponentially with time at a rate which depends principally on the local shear rate. In one case, the velocity gradient field is known a priori, and the measurements serve to verify the utility and accuracy of the method. In the other, we study the experimentally important 'four roller' flow device where the velocity gradient field was only approximately known before the present investigation. Additional experiments were also performed in order to investigate the effects of smaller values of the time-scale ratio, $\tau_{t} / \tau_{s}$, illustrating a situation where the theory presented would not be applicable. These experiments also suggest the necessary conditions for the effects of diffusion to be measurable in the presence of flow.

The experiments were performed on two separate flow devices: the four-roll mill, illustrated in figure 3, which is used to approximate the two-dimensional, hyperbolic flow of equation (17); and a concentric cylinder, 'Couette' device, shown in figure 4, which approximates the simple, shear flow of equation (16). The light source for both experiments was an argon-ion laser operating typically in the neighbourhood of $500 \mathrm{~mW}$, and the scattered light was detected using an EMI 9789 photomultiplier tube. Two pinholes separated by about 10 inches were used to collimate the scattered light and thereby define the scattering volume. The pinholes had a diameter of $\frac{1}{32}$ in. in the four-roll experiment, and $\frac{1}{64}$ in. in the case of the Couette device, and the incident wavelengths of the light were 5145 and $4880 \AA$, respectively. The output of the photomultiplier tube was amplified by a Princeton Applied Research 113 preamplifier and processed with a Saicor 43A 400-channel correlator. The computed correlation function was then output onto a Hewlett Packard $700 \mathrm{~A} X-Y$ recorder.

The dimensions of the four-roll mill are shown in figure 5. All rollers were driven separately with independent motors and each could be removed to allow for a one- 


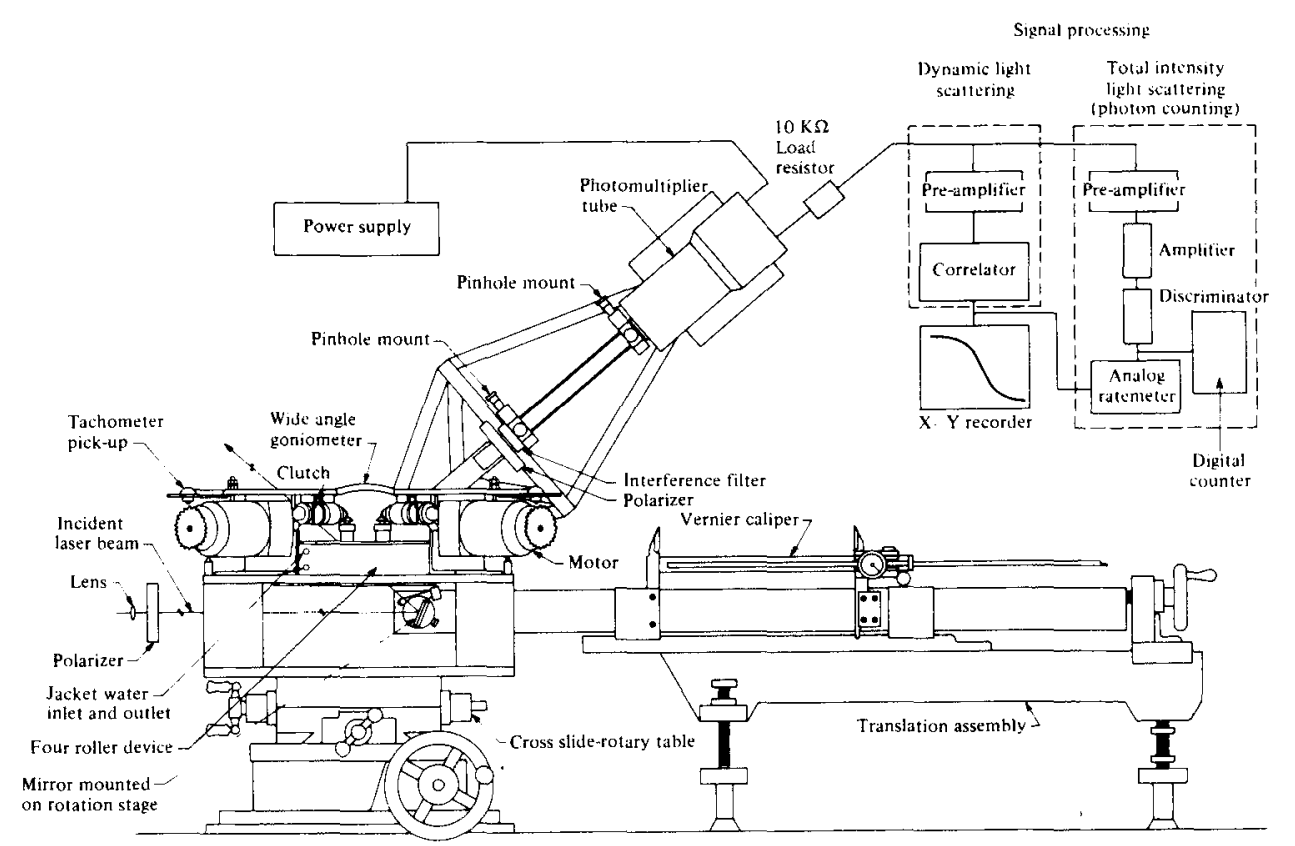

Frgure 3. Light-scattering set-up with the four-roll mill.

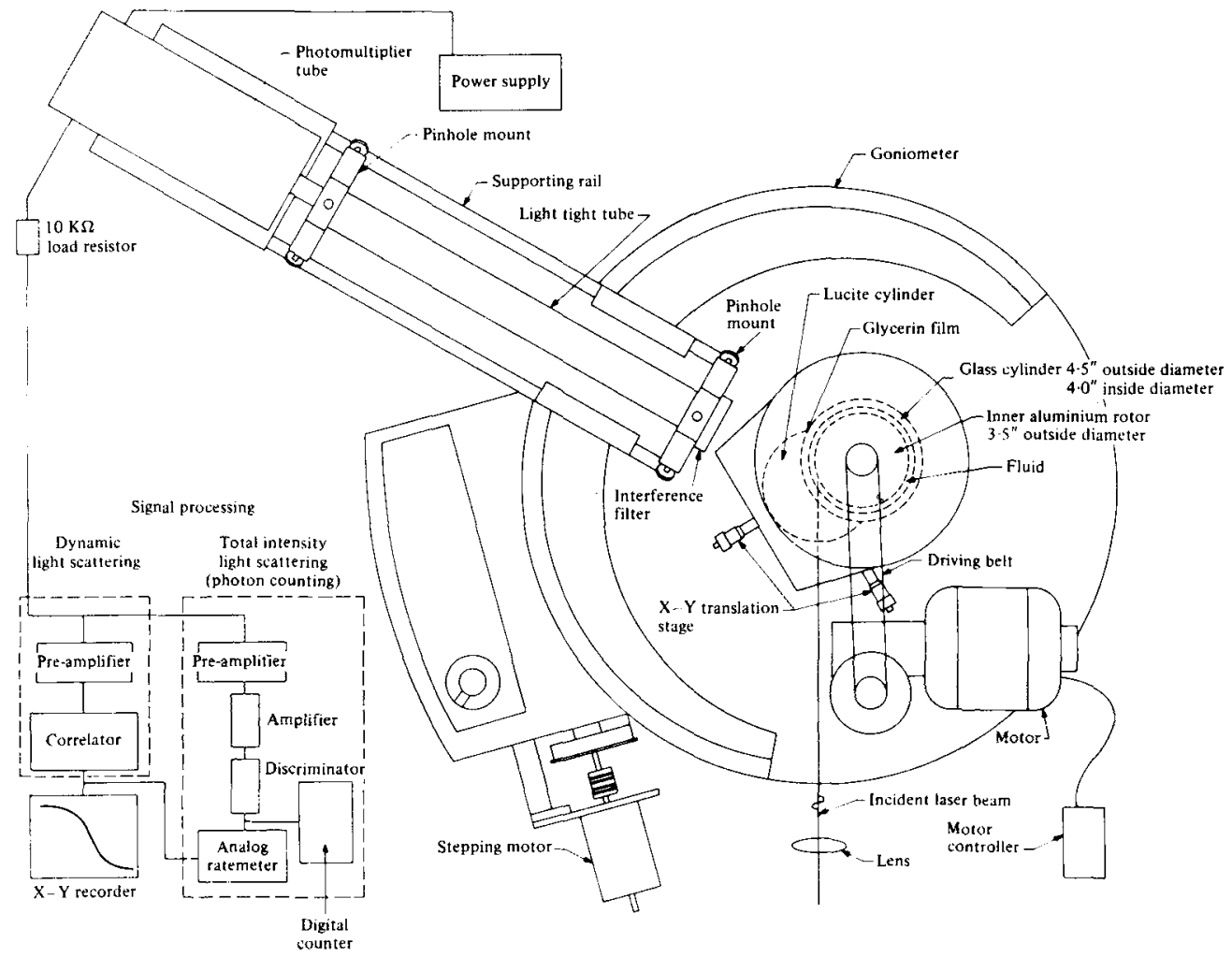

Fiaure 4. Light-scattering set-up with the Couette apparatus. 

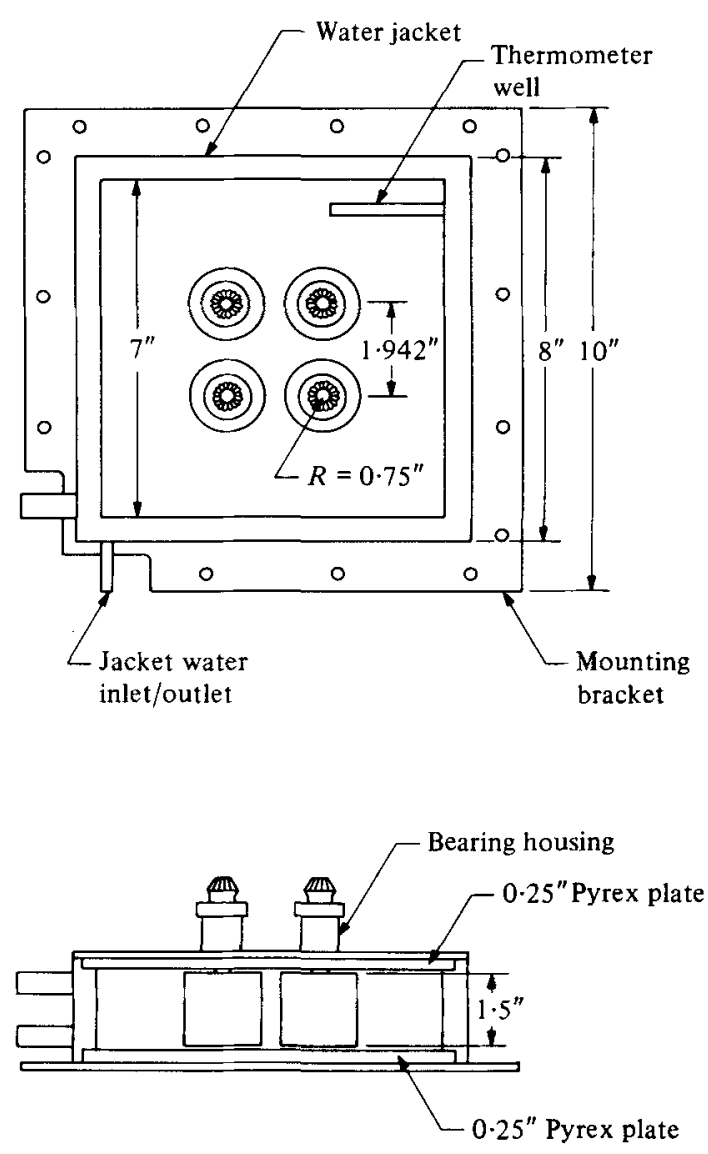

Figure 5. The four-roll mill.

or two-roller operation. The top and bottom of the container were constructed of $\frac{1}{4}$ in. Pyrex through which the laser beam passed. A photographic study of the flow field generated by the apparatus was undertaken using a dilute suspension of glass spheres of mean diameter $100 \mu \mathrm{m}$ in glycerin. A typical streakline photograph of the centreplane velocity field is shown in figure 6 , from which it appears that the two-dimensional hyperbolic flow of equation (17) is reasonably well approximated in the central region between the four rollers. Assuming this to be true, the (uniform) velocity gradient in this region can be obtained by measuring the travel time $\Delta t$ for a tracer particle to move from position $\left(x_{1}, y_{1}\right)$ to $\left(x_{2}, y_{2}\right)$. The velocity gradient for a two-dimensional hyperbolic flow is

$$
\gamma \equiv \ln \left(\frac{x_{2}}{x_{1}}\right) / \Delta t=\ln \left(\frac{y_{1}}{y_{2}}\right) / \Delta t
$$

This procedure $\dagger$ was carried out for rotation rates, $w$, of the rollers ranging from $\mathbf{1 . 4}$ to $35 \mathrm{~s}^{-1}$. The result was a linear relationship between roller speed and apparent velocity gradient,

$$
\gamma=0.678 w \mathrm{~s}^{-1},
$$

$\uparrow$ Further details are reported by Fuller (1980). 
as would be expected if the flow were truly given by equation (17). An interesting question, which we shall investigate later in this section, is whether detailed pointwise measurements of the velocity gradient field would support the conclusion of a very good approximation to the hyperbolic flow which is the obvious and strong inference of our streakline visualization studies. In the light-scattering experiment in the four-roll mill, the incident beam was directed into the flow device through its bottom by means of a front-surfaced mirror, mounted on a rotation stage capable of $360^{\circ}$ orientation to within $0 \cdot 25^{\circ}$. The rotation stage was in turn mounted on a translation stage capable of a twelve inch traverse to within $0.001 \mathrm{in}$. Angular variation of the scattered-light wave vector was provided by mounting the photomultiplier tube on a wide-range vertical goniometer which could be positioned to within $0.01^{\circ}$. This capability of orientating the incident and scattered wave vectors separately allows the orientation and magnitude of the scattering vector $\mathbf{q}$ to be varied independently. In addition, the four-roll mill itself was mounted on a cross-slide rotary table which provides the capability of translating and rotating the flow device, while holding the optics stationary. Thus, scattering experiments at different positions and orientation relative to the flow are possible.

The Couette device was constructed of a rotating inner cylinder of black, anodized aluminium, enclosed by a heavy-walled, precision-bore glass tube which was held stationary. The dimensions of the various components are indicated in figure 4 . In this case, the incident laser light was introduced into the gap between the two cylinders through a solid lucite cylinder which was cut to fit on to the outer glass cylinder such that the composite glass plus lucite wall formed a cylindrical section with its central axis at the centre of the gap. This enabled the incident beam to enter the gap region with a minimum of refraction at the lucite/air interface. The Couette device was itself mounted on an $X-Y$ translation stage which, in turn, was fastened to the hub of a horizontal goniometer. The goniometer hub could be rotated independently of the rotating arm which supported the photomultiplier tube.

Experiments in both apparatus were conducted using a suspension of 100 p.p.m. weight of Dow Polystyrene Latex spheres in glycerin. The particle diameter was $0.091 \mu \mathrm{m}$ and the viscosity of the glycerin was found to be 7 poise at $20^{\circ} \mathrm{C}$. The water content of the glycerin was not known.

In order to use equation (21) to analyse the experiments, it is necessary to ensure that the dynamics of the light-scattering process occur primarily on the shear-rate time scale $(q \gamma L)^{-1}$. The data necessary to determine the various time-scale ratios for the two experiments are listed in table 1 . In table 2 we show the corresponding timescale ratios, $\tau_{t} / \tau_{s}, \tau_{D} / \tau_{s}$ and $\tau_{f} / \tau_{s}$. It may be seen, in both cases, that the time scale $\tau_{s}$, associated with the mean velocity gradient, is very much the smallest, and it is thus expected that equation (21) should adequately represent the measured correlation functions.

To obtain absolute values for the shear rate from (21) and the measured correlation function, it is necessary to also measure the intensity profile of the beam, as we have already noted. However, this was not done in the present experiments. Instead, the characteristic time, in each instance, was estimated as the time for decay of the correlation function to one-half its initial magnitude, and the shear rate was taken as being proportional to the inverse of this time. Thus, the velocity gradients obtained (and reported) are all relative values. 


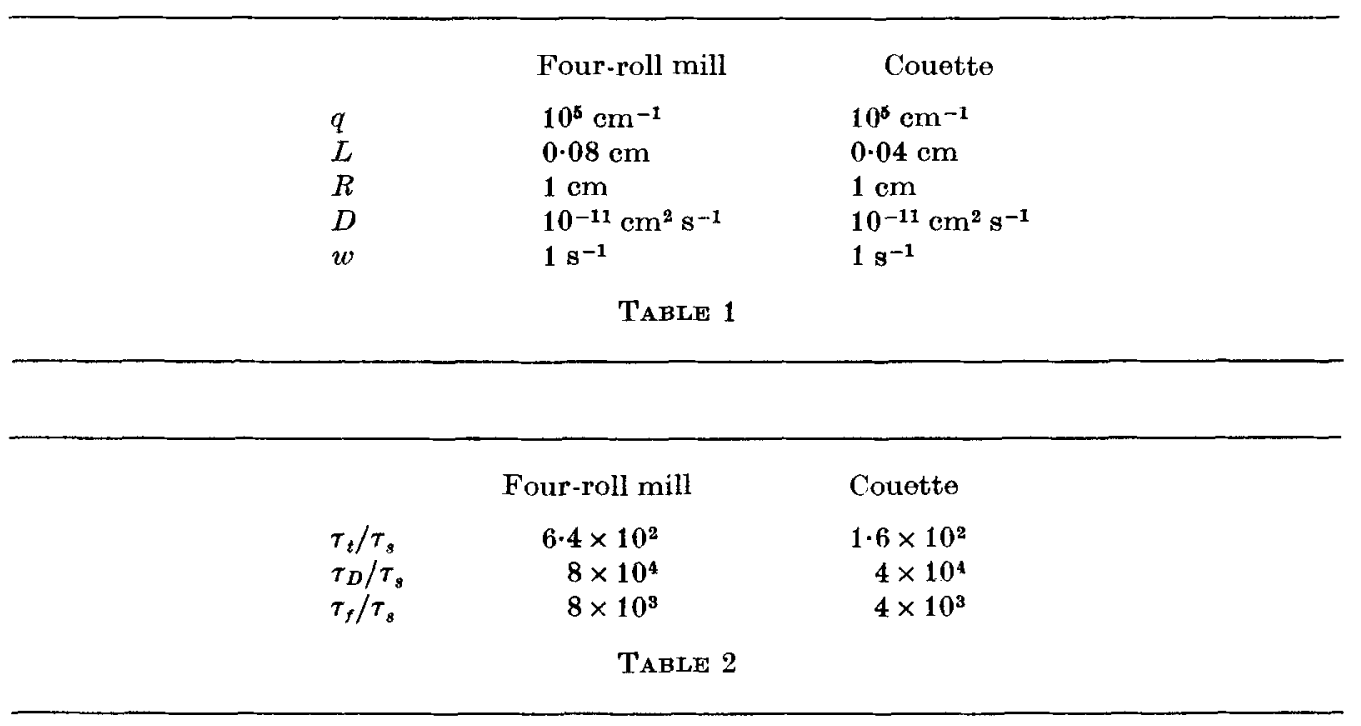

The first flow field studied was that generated by a single rotating cylinder and this was accomplished by removing all but one of the rollers in the four-roll mill. Analysis of an infinitely long cylinder of radius $R$, rotating with angular speed $w$, yields an $r^{-2}$ dependence of shear rate on distance from the cylinder centre

$$
\gamma=-w R^{2} / r^{2}
$$

Measurement of the spatial dependence of the velocity gradient should thus lead to the same radial dependence, provided that the flow device approximates an infinitely long cylinder.

The experimental arrangement for this case is shown in figure 7. The scattering vector $\mathbf{q}$ was orientated in the azimuthal direction and the experiment was carried out by holding the optics stationary while translating the flow device along the path indicated in figure 7. At each interval of translation, the correlation function was obtained and recorded. A typical correlation function is pictured in figure 8 . The results of this procedure for two-roller speeds are shown in figure 7 where we have plotted the measured velocity gradient against the square of the reciprocal of the radial distance from the roller centre. The linearity of the data, when plotted in this fashion, indicates that the measured velocity gradient is consistent with the theoretical expression (32). Furthermore, the fact that the slope doubles when $w$ is doubled is also consistent with (32). Both of these results support the validity of the homodynescattering method as a way of measuring the velocity gradient.

The data do depart from linearity farther from the roller surface, but we attribute this to boundary effects associated with the finite cylinder length. The presence of a top and bottom on the four-roller container causes the velocity gradient to drop off faster than the analysis for an unbounded fluid would indicate. It is important to note that throughout the experiment, the shapes of the correlation functions were observed to remain constant. That is, by scaling the time, the correlation functions could be made to trace out a single curve. This verifies the assertion that the only 


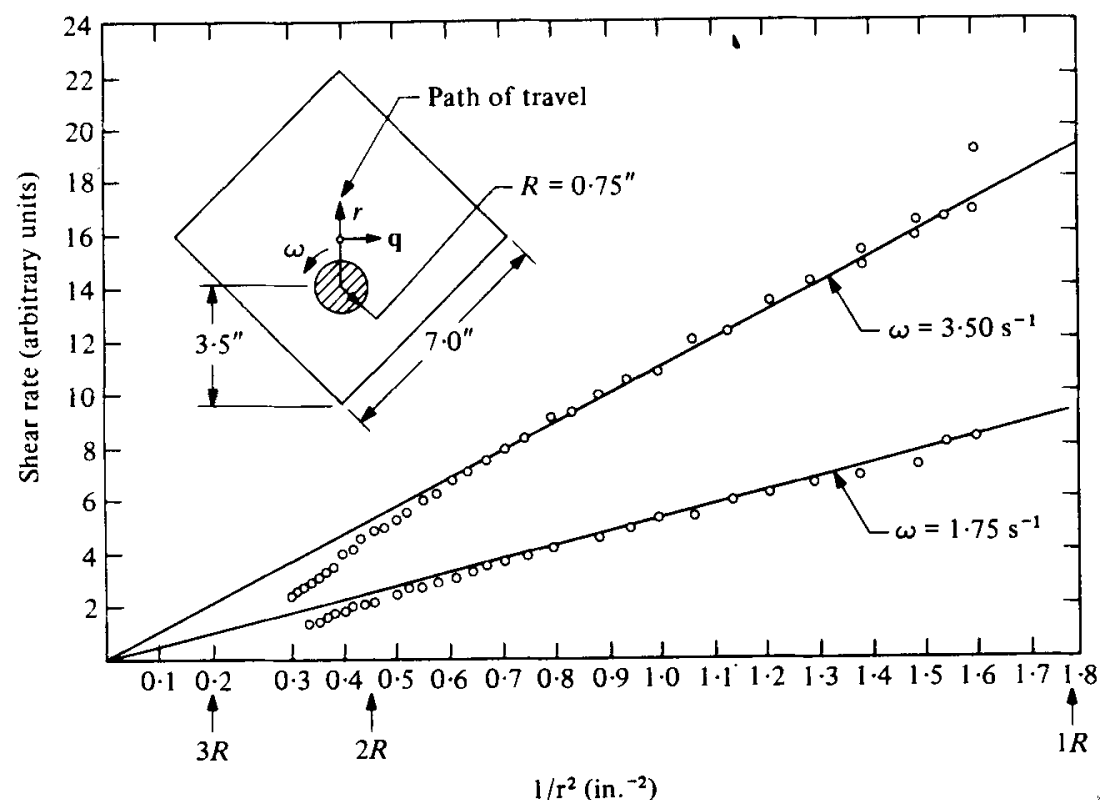

Figurs 7. Measured shear rate as a function of the radial distance from a single rotating roller.

The illustration in the upper left-hand corner represents the experimental arrangement.

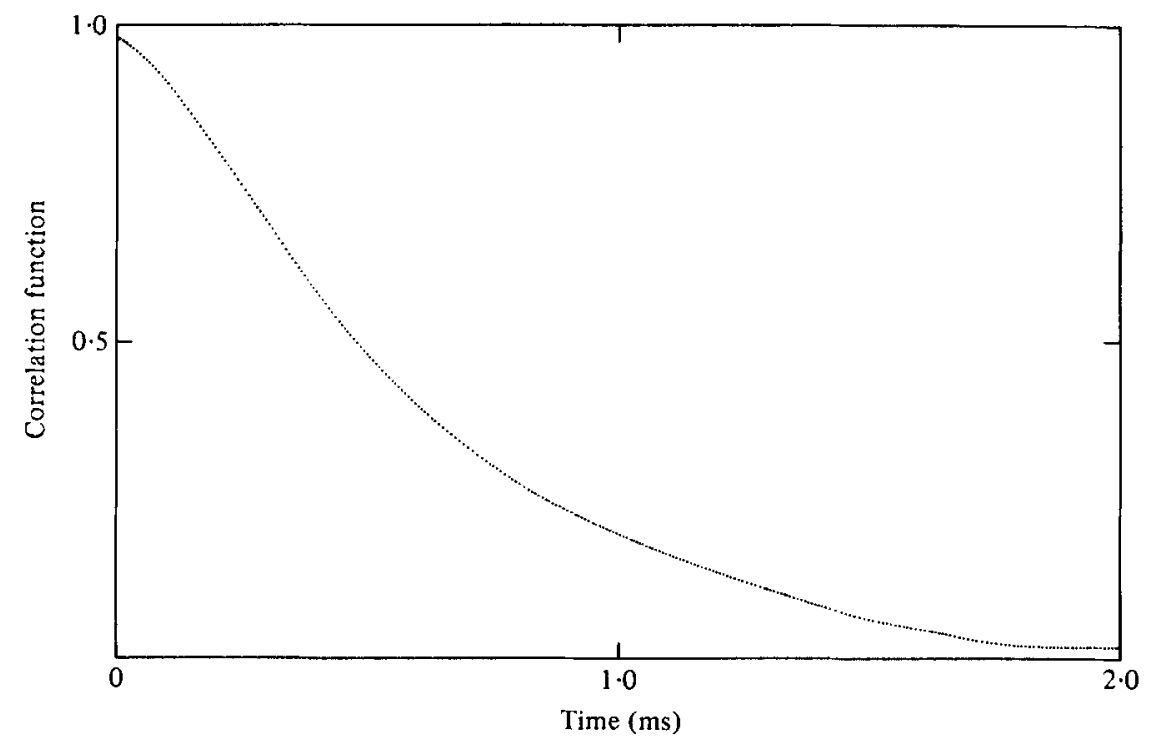

Figure 8. Typical correlation function for the single rotating roller experiment. Data taken at a scattering angle of $30^{\circ}$.

quantity changing is the velocity gradient and that there is only a single time scale controlling the correlation function.

A second experiment was conducted using the Couette device, again intended to verify the homodyne method for measuring velocity gradients. In this apparatus, the flow field is a good approximation to simple shear flow and the velocity gradient should 


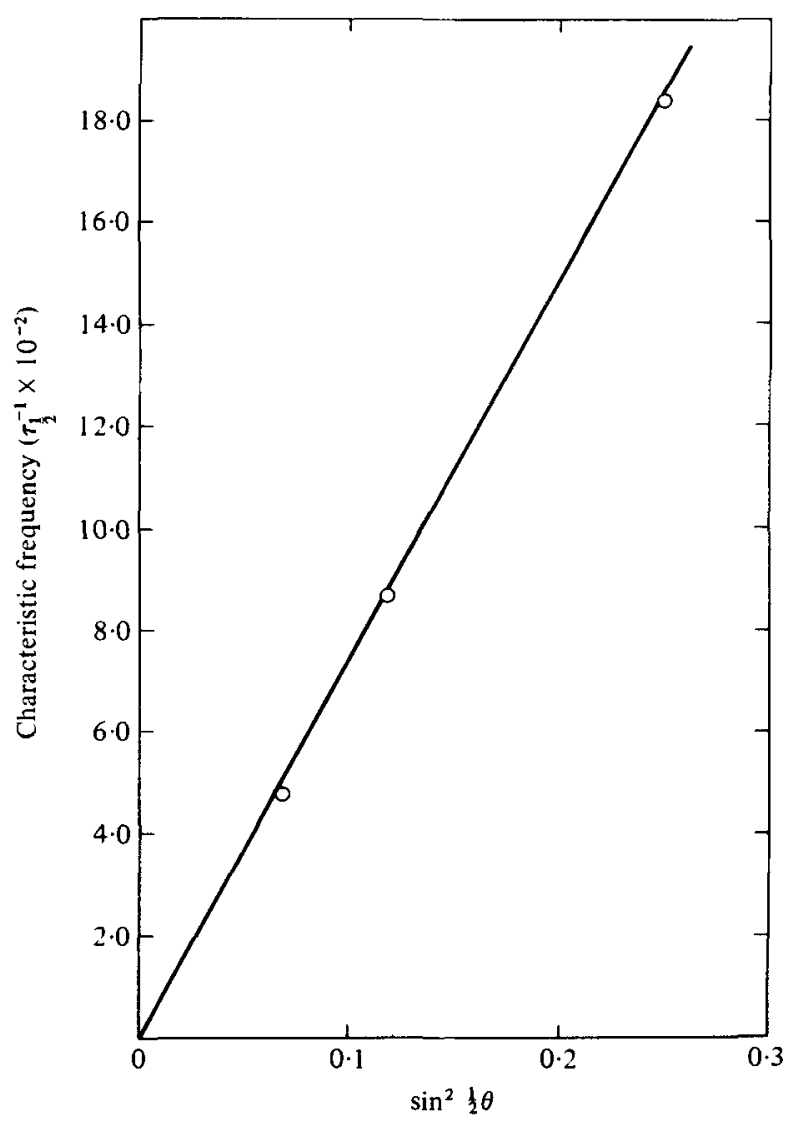

Figure 9. Characteriatic frequency of the homodyne correlation function $v s$. $\sin ^{2} \frac{1}{2} \theta$. The flow is simple shear with the direction of flow parallel to the incident beam vector.

thus be nearly constant throughout the gap. The incident wave vector $\mathbf{k}_{i}$ was kept parallel to the direction of flow and correlation functions were obtained as a function of scattering angle. Care was taken to ensure that the centroid of the scattering volume remained fixed as the scattering angle was varied.

Taking the velocity to be locally in the direction of $x$ and the gradient to be in the direction of $y$, the quantity q. $\boldsymbol{\Gamma}$. xt would be $2 \gamma\left|\mathbf{k}_{i}\right| \sin ^{2} \frac{1}{2} \theta y t$. Therefore, the measured characteristic decay time for the $z$ correlation function should be linear with respect to the quantity $\sin ^{2} \frac{1}{2} \theta$. The data plotted in figure 9 show that this relationship is satisfied, thus again confirming the accuracy of the method for measurement of the velocity gradient.

Finally, as an indication of the kind of study which can be done in more complicated flows, we have used the homodyne-scattering method to determine the velocity gradient profile for the four-roll mill when it is supposed to be generating hyperbolic flow. These data are of some intrinsic interest as the four-roller device has often been used in studies of particle and droplet deformation and orientation but has previously been characterized only via visual, streakline photography $\dagger$ (Pope \& Keller 1977;

$\dagger$ A more comprehensive study of the four-roller device is reported by Fuller (1980). 
Rumscheidt \& Mason 1961; Taylor 1934). The four-roller device is usually designed to simulate two-dimensional extensional flow and it is important to know how well the device performs in actually creating this type of homogeneous flow. The most important feature of the flow, for most of the particle dynamics experiments, is the absence of spatial variation of the velocity gradient field.

As discussed in $\$ 2$, the scattered light spectrum will generally be influenced by all non-zero gradient components of the projected velocity v.q. However, for the case of a single rotating cylinder, with the light-scattering volume situated half way between the top and bottom of the cylinder, there is, at leading order, only a single, radial component of the velocity gradient, and there is therefore no ambiguity in interpreting what the experiment measures. However, in the more complex geometry of the four-roll mill, there will in general be more than one non-zero component of the velocity gradient. By making the scattering volume highly elongated one could, in principle, determine each velocity gradient component separately. We did not attempt this procedure here, but instead used symmetry of the flow field at the centre plane and took measurements along the centre streamline of the centre plane with the scattering vector $\mathbf{q}$ parallel to the velocity component in the $x$ direction. Along this centre streamline, $\partial v_{x} / \partial y$ and $\partial v_{x} / \partial z$ are zero to at least a first-order of approximation, and the experiment thus yields values for $\partial v_{x} / \partial x$.

It may be anticipated that the velocity field in the four-roll mill contains a saddle point close to the position of closest approach between any two adjacent rollers. At these points the velocity gradient will vanish and the time-scale ratio $\tau_{t} / \tau_{s}$ will no longer be large enough for the correlation function to be related to the intensity profile by equation (20). Therefore, in the vicinity of these points one would expect the correlation function to change shape and to no longer be characterized by a single time scale which is inversely proportional to the velocity gradient. It was found, however, that the regions where there was a noticeable change in the shape of the measured correlation function were very small (less than one-tenth of an inch in the flow direction) and data in these regions are simply not included here.

The measured velocity gradient profile is pictured in figure 10. Data taken with the direction of the rollers reversed fell on the same curve. The profile clearly shows that the region of homogeneity in the flow field (constant velocity gradient) is confined to an area whose length is approximately equivalent to the gap width between the rollers. A study was also undertaken of the dependence of the velocity gradient on roller speed with the scattering volume stationary at the centre of the four-roll device. This study showed that the dependence was linear over the entire range of roller speeds $\left(0-35 \mathrm{~s}^{-1}\right)$.

A short investigation was also carried out in order to see whether conditions could be achieved where it would be possible to see the effects of diffusion of the scattering particles on the scattering data. Such data would be of considerable interest since the rate of diffusion is sensitive to the conformation of the scattering particles (or molecules) and would provide information about flow-induced deformation or, in the case of rigid non-spherical particles, orientation. This study was carried out in both apparatus and involved an attempt to set the scattering vector $q$ normal to the plane of flow so that q. $\boldsymbol{\Gamma} . \mathbf{x} \equiv 0$ everywhere in the scattering volume. In the Couette device, the vector $q$ was orientated radially with a scattering angle of about $60^{\circ}$. In the four-roll mill experiment, $\mathbf{q}$ was set parallel to the $z$ axis with a scattering angle 


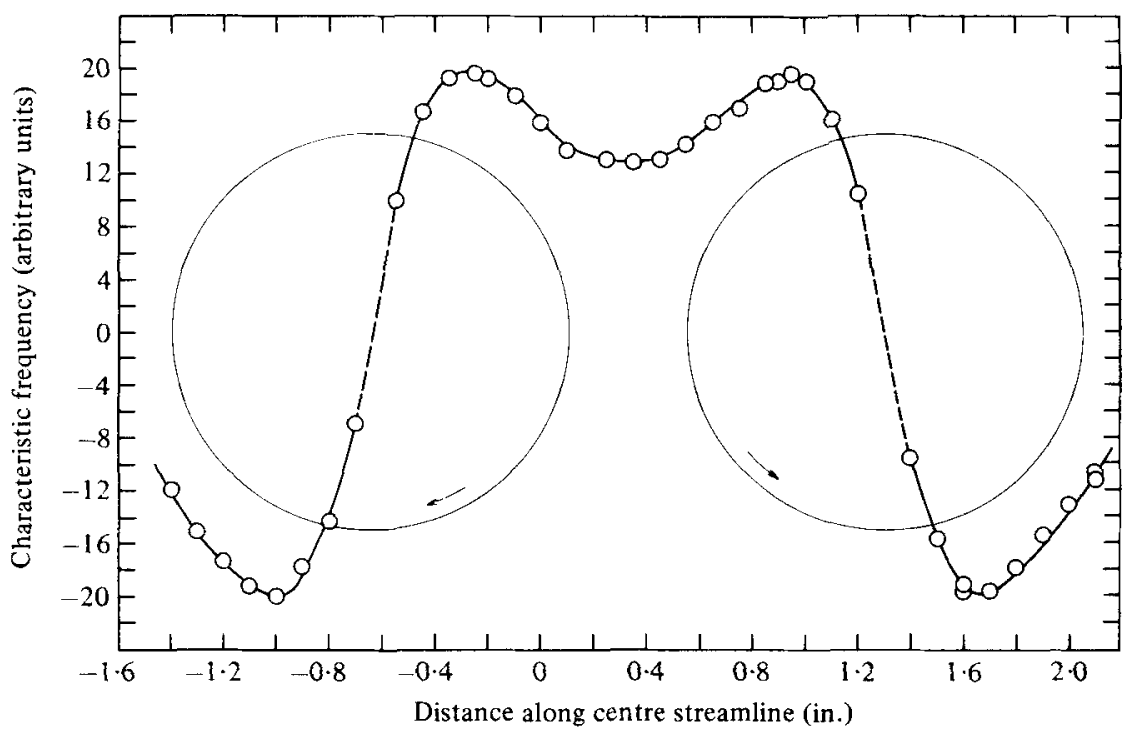

Figure 10. Characteristic frequency as a function of distance along the centre stream line in the four-roll mill simulating hyperbolic flow. The scattering angle was $50 \cdot 7^{\circ}$ and the rate of rotation of the rollers was $1.75 \mathrm{~s}^{-1}$. The large circles indicate the position of the rollers with respect to the bottom ordinate.

of $120^{\circ}$. If equation (15) was appropriate and the orientation of $q$ sufficiently precise, this configuration would eliminate the effect of the shear rate on the light scattering correlation function, leaving only the diffusion process. However, in the case of the Couette apparatus, the presence of a small but finite curvature in the streamlines and a non-zero mean velocity in the scattering volume caused the correlation function to be dominated still by flow effects and the measured time scale for the correlation function remained orders of magnitude faster than the diffusive time scale. In the four-roll mill, on the other hand, the measured characteristic time scale was reduced to the same order of magnitude as the diffusive time scale. This was accomplished by placing the scattering volume in the vicinity of the stagnation point in the flow and using a high scattering angle. There was, however, a small $z$ component of velocity and an associated velocity gradient due to the effect of the top and bottom boundaries, and for this reason it would probably not be possible for the diffusive process to completely dominate the correlation function in the present system.

The criterion which must be satisfied in order that the diffusive effects dominate the spectrum is that

$$
\tau_{t} / \tau_{D}=\frac{q D^{2} L}{U} \gg 1
$$

If the scattering volume is centred on the stagnation point, the velocity $U$ will be of the order of $\gamma L$ and the ratio becomes

$$
\tau_{t} / \tau_{D}=\frac{q^{2} D}{\gamma}=\frac{(q l)^{2}}{P e}
$$

For most situations of interest, the Péclet number will be no larger than $O(1)$. Therefore, in order to exhibit a dominant diffusive effect, the experiment would have to be 
performed at higher angle using relatively large particles. It would also be necessary, as in the present experiments, to have the scattering vector normal to the plane of flow and to have the scattering volume centred on a stagnation point.

\section{Conclusions}

We have examined the application of homodyne light scattering techniques to a laminar flow field, both theoretically and experimentally. Although many dynamic processes contribute to the frequency spectrum of the scattered light, we have seen that the dominant effect on the light scattering correlation function will normally be due to the presence of velocity gradients in the scattering volume. Thus, we have shown theoretically, and confirmed via experiment, that the homodyne light scattering experiment provides a direct method to measure the velocity gradients.

In its present state of development, the most serious limitation of the method is that all components of the gradient of the projected velocity, v.q, contribute to the spectrum. Although this deficiency may be overcome in principle by creating an anisotropic scattering volume, this was not pursued in the present investigation since the experiments studied had only one gradient component of velocity in the scattering volume. A second limitation is that the intensity profile of the beam must be known in order to quantify the gradients in an absolute sense. This information can be obtained experimentally without much difficulty. Unfortunately, however, the intensity profile will generally not be maintained for extended periods of time. The laser beam profile in our system was certainly stable for periods of the order of days, but often changed in as little as a week as the laser optics require cleaning and retuning.

The chief advantage of the homodyne light scattering technique relative to more conventional measurement techniques is that the shear rate can be obtained in a single, 'non-invasive' experiment, without the need for accurate repositioning of the measurement point as would be required if one were to simply differentiate velocity data. The results are therefore more accurate and reliable than was previously possible. In addition, the present technique can be used in situations where the velocity changes rapidly in narrow regions (and in this case, the principal component of $\nabla \mathbf{u}$ is normal to $\mathbf{u}$, thus minimizing the major ambiguity of interpretation which was discussed above).

In contrast, the usefulness of homodyne scattering experiments to determine anything of the diffusive motions (and, thus, indirectly the conformational state) of the scattering elements as is conventionally done in quiescent solutions or suspensions, would appear to be highly limited for flowing systems. The short investigation which we have carried out here suggests that it would be necessary for the scattering volume to be centred on a stagnation point of the flow, in order even to approach the necessary conditions for the correlation function to be dominated by the effects of Brownian diffusion. In addition, in order that $\tau_{D} / \tau_{t}<O(1)$, it is necessary that $q l>O(1)$ and this requires that the measurements be carried out at high scattering angles with relatively large particles. These various restrictions greatly reduce the potential utility of homodyne dynamic scattering for purposes other than measurement of the local mean velocity gradient in flowing systems.

This work was supported by a grant from the Office of Naval Research. 


\section{REFERENCES}

Berman, N. S. \& Dunning, J. W. 1973 Pipe flow measurements of turbulence and ambiguity using laser-Doppler velocimetry. J. Fluid Mech. 61, 289.

Berne, B.J. \& Pecora, R. 1976 Dynamic Light Scattering with Applications to Chemistry, Biology, and Physics. Wiley.

Bourke, P. J., Butterworth, J., Drain, L. E., Egelstaff, P. A., Hughes, A. J., Hutchingon, P., Jackson, D. A., Jakeman, E., Moss, B., O'Shavghnessy, J., Pike, E. R. \& Schofield, P. 1970 A study of the spatial structure of turbulent flow by intensity-fluctuation spectroscopy. J. Phys. A 3, 216.

Edwards, R. V., Angus, J. C., French, M. J. \& Dunnina, J.W. 1971 Spectral analysis of the signal from the laser-Doppler flowmeter: Time independent systems. J. Appl. Phys. $42,837$.

Fuller, G. G. 1980 Ph.D. thesis, California Institute of Technology.

PIKE, E. R. 1976 Photon correlation velocimetry. In Photon Correlation Spectroscopy and Velocimetry (ed. H. Z. Cummins \& E. R. Pike). Plenum.

POPE, D. P. \& KELLER, A. 1977 Alignment of macromolecules in solution by elongational flow. J. Colloid \& Polymer Sci. 255, 633.

Rumscheidt, F. D. \& Mason, S. G. 1961 Particle motions in sheared suspensions. XI. Internal circulations in fluid droplets. J. Colloid Sci. 16, 210.

TAYLOR, G. I. 1934 The formation of emulsions in definable fields of flow. Proc. Roy. Soc. A $146,501$. 



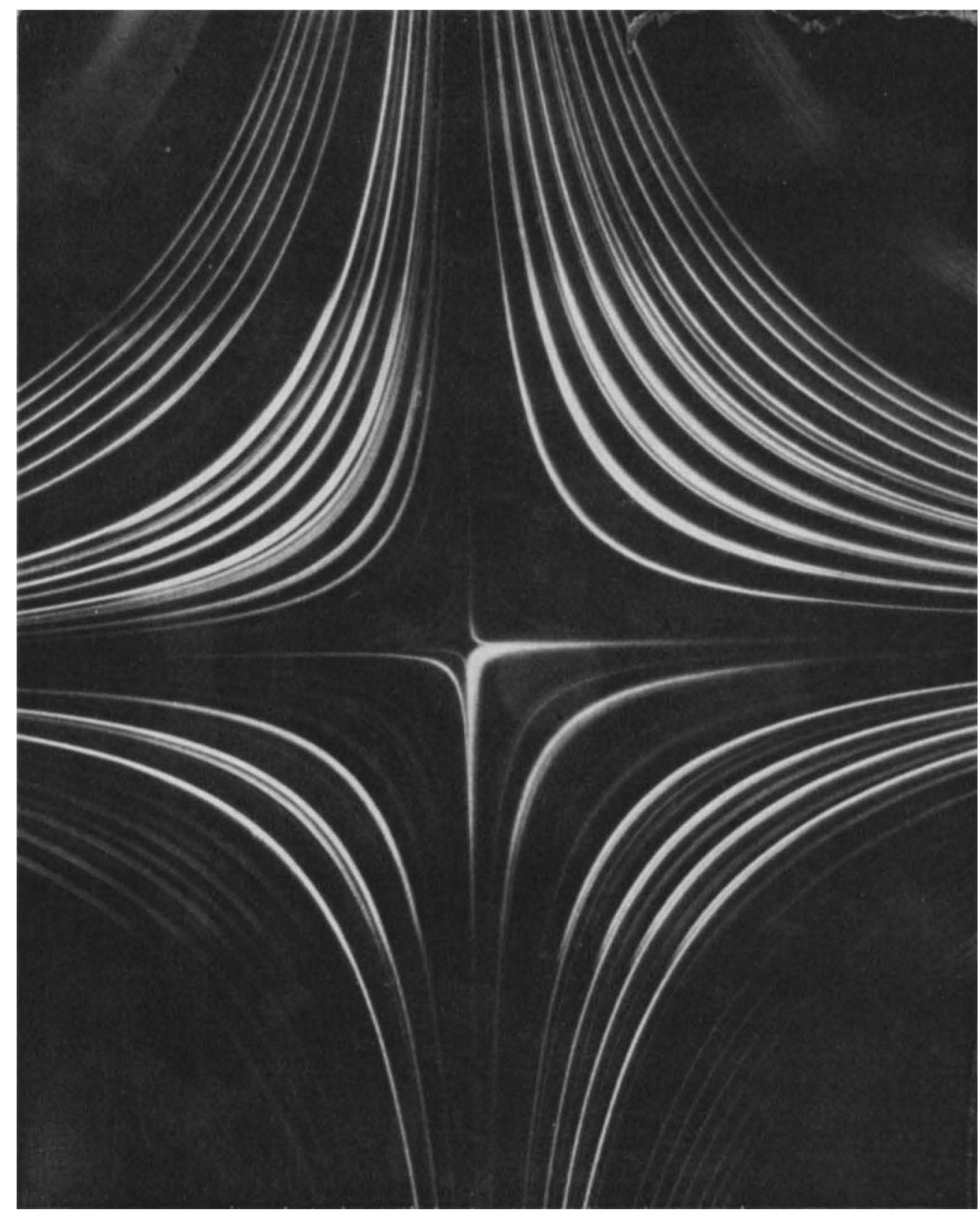

FIGURE 6. Streakline photograph of flow generated by the four-roll mill. The particles wore illuminated by a planar sheet of light at the mid-height of the rollers. 